\title{
Comparison of different predictive tests for difficult airways in pediatrics
}

\author{
Yeliz Kılıç ${ }^{1}$, Meryem Onay ${ }^{1}$, Dilek Çetinkaya ${ }^{1}$, Ayten Bilir ${ }^{1}$, Birgül Büyükkıdan Yelken ${ }^{1}$ \\ 1 Department of Anesthesiology and Reanimation, Faculty of Medicine, Osmangazi University, Eskişehir, Türkiye
}

Yeliz Kılıç, ORCID: 0000-0003-1446-7747

Meryem Onay, ORCID: 0000-0002-5028-9135

Dilek Çetinkaya, ORCID: 0000-0002-9320-7005

Ayten Bilir, ORCID: 0000-0002-3491-3209

Birgül Büyükkıdan Yelken, ORCID: 0000-0001-9677-9028

\begin{abstract}
Objective: There are few studies regarding the use of predictive tests for difficult airways in pediatrics. The aim of this study was to investigate the effectiveness of the modified Mallampati test (MMT), the upper lip bite test (ULBT) and anthropometric measurements of the head and neck in the prediction of difficult airways in children.

Methods: Forty-eight pediatric patients who underwent elective surgery under general anesthesia with endotracheal intubation were recruited for the study. During the preanesthetic evaluation, airway status was evaluated using three methods: MMT, ULBT and anthropometric measurements of the head and neck. Laryngoscopy was performed with a single blade of a videolaryngoscope and airway status was evaluated using the Cormack-Lehane classification. All patients were then classified into two groups: difficult airway or easy airway.
\end{abstract}

Results: Ten (20.8\%) patients were classified as the difficult airway group. In this group, $80 \%$ of the patients had MMT grade 3-4 $(p=0.001)$ while $50 \%$ of the patients were classified as ULBT $3(p=0.000)$. Interincisor distance (ID), hyomental distance (HMD), distance from ear tragus to the corner of the mouth (DTM) and distance from frontal plane to chin (DFC) were significantly different between the groups $(p<0.05)$. In ROC curve analysis, ULBT had the largest area under the curve (AUC=0.880).

Conclusion: ULBT, MMT, HMD and ID were the most consistent predictors of difficult airway status. ULBT was superior to other tests because of its larger AUC and higher sensitivity and specificity rates. However, difficulty in applicability in young children seemed to be the most important limitation of both ULBT and MMT.

Keywords: Airway management, laryngoscopy, pediatrics. 


\section{Introduction}

Airway control is one of the most important steps in pediatric anesthesia management. Compared to adults, children have a narrower airway associated with a higher risk of edema due to procedures such as endotracheal intubation. Approximately $13 \%$ of perioperative respiratory problems are related to difficulties during intubation. ${ }^{[1]} \mathrm{Ad}-$ ditionally, difficulties in airway management can result in serious conditions such as cardiac arrest, brain damage and even death. ${ }^{[2,3]}$

To date, observational methods such as the modified Mallampati test (MMT) and the upper lip bite test (ULBT), anatomical measurements including thyromental, hyomental and sternomental distances, and scoring systems such as the Naguib score have been recommended to predict difficult intubation in adult patients. ${ }^{[4-6]}$ However, there are a limited number of studies in the literature regarding the use of such diagnostic tests in the pediatric population. ${ }^{[7-11]}$

In the present study, compared with the Cormack-Lehane classification, the effectiveness of widely used bedside tests including MMT and ULBT and anthropometric measurements of the head and neck was examined to predict difficult airway status in pediatric patients under general anesthesia.

\section{Materials and Methods}

\section{General Data}

After receiving approval from the local ethics committee (2019/48), this prospective observational study was conducted in the Osmangazi University Hospital between January and November 2020, in accordance with the Declaration of Helsinki. Pediatric patients with American Society of Anesthesiologists (ASA) physical status 1-3 who were scheduled to undergo elective operations under general anesthesia with endotracheal intubation were recruited for the study. Patients who had any head-neck tumor or headneck anomaly were excluded from the study. Patients were selected for the study using nonprobability sampling. Parents were preoperatively informed about the study and in- formed consent forms were obtained. Patient demographic data including age, gender, weight, height and all anesthetic techniques were recorded.

\section{Preanesthetic evaluation of airway status}

During the preanesthetic evaluation of the patients, airway status was evaluated using three methods: MMT, ULBT and anthropometric measurements of the head and neck. In the preanesthetic evaluation, oropharyngeal structures were observed and recorded in a sitting position with the mouth wide open and tongue out, according to the Mallampati classification modified by Samsoon and Young. ${ }^{[4]}$ Patients with Mallampati grade 3-4 were classified as candidates for difficult intubation.

Airway status was also evaluated with the ULBT. ${ }^{[12]} \mathrm{Al}-$ though this test can be evaluated more clearly in patients over six years old, data of younger children who comply with the test were also used. ULBT is based on the ability to bite the upper lip with the lower incisors: ability to bite the upper lip above the vermillion (grade 1), ability to bite under the vermillion (grade 2) and inability to bite the upper lip (grade 3). Grades 1-2 were accepted as easy intubation, while grade 3 was considered as a candidate for difficult intubation.

In addition, seven anthropometric measurements (NC, ID, TMD, SMD, HMD, DFC and DTM) were also recorded.

\section{Anesthesia management}

No premedication was allowed and the anesthetic technique was the same for all patients. In the operating room, the patients were positioned with pillows under the head and the neck was extended. Heart rate, blood pressure, $\mathrm{SpO}_{2}$ and end tidal carbon dioxide were continuously monitored. Following 5-8\% sevoflurane induction for all patients with $50 \% \mathrm{O} 2$ and $50 \%$ air, standard induction was performed with lidocaine $\left(1 \mathrm{mg} \mathrm{kg}^{-1}\right)$, propofol $(2-4 \mathrm{mg}$ $\left.\mathrm{kg}^{-1}\right)$ and remifentanyl $\left(0.5 \mu \mathrm{g} \mathrm{kg}^{-1}\right)$. Rocuronium $(0.5 \mathrm{mg}$ $\left.\mathrm{kg}^{-1}\right)$ was intravenously administered for neuromuscular relaxation after mask ventilation. The laryngoscopic view was rated with the patient's head in the sniffing position 
without external laryngeal manipulation. Endotracheal intubation and evaluation of difficulty of laryngoscopy was performed by the same experienced anesthesiologist.

Laryngoscopy was performed with a single laryngoscope blade of a videolaryngoscope and airway status was evaluated using the Cormack-Lehane classification. ${ }^{[13]}$ Grade 3 and 4 were classified as difficult airways whereas grade 1 and 2 were accepted as easy airways. All patients were then classified into two groups: patients with difficult airways and those with easy airways.

\section{Statistical evaluation}

A power analysis (using the Chi-square method) based on a similar previous article showed that a sample size of 42 patients was required to achieve a power of $95 \%$ with a significance level of $5 \%$ for evaluating differences between the groups with or without difficult airway status according to the Cormack-Lehane classification. ${ }^{[14]}$ The standard version of Statistical Package for the Social Sciences (SPSS 23.0 software) was used for statistical analysis. Descriptive analyses are presented as number/(\%) and mean \pm SD/ (\%) for categorical and continuous variables, respectively. Differences between the two groups were evaluated using Chi-square, Mann Whitney U and Fisher's exact tests. The predictive abilities of all preoperative tests (their ability to predict difficult airways) were evaluated by ROC curve analysis and compared with each other. $\mathrm{P}$ value less than 0.05 was accepted as significant.

\section{Results}

\section{Baseline characteristics}

Forty-eight pediatric patients with a mean age of 6.1 years (1-14) were included in the study. There were 27 $(56.2 \%)$ males and 21 (43.8\%) females. The majority of patients were classified as ASA 1 (32, 66.7\%) and ASA 2 $(13,27.1 \%)$ preoperatively. In the preanesthetic evaluation, $24(50 \%)$ patients had grade 1 or 2 Mallampati score while $13(27.1 \%)$ were classified as Mallampati 3 or 4 . The remaining 11 patients under five years old were not classified because of the inability to perform the Mallampati test.
Similarly, the ULBT test could not be performed for 15 (31.3\%) small children. Twenty-six patients were classified as class 1 or 2 whereas the remaining 7 patients were assessed as class 3 , according to the ULBT.

\section{Comparison of baseline data and predictive tests}

Ten (20.8\%) patients were classified as the difficult airway group while $38(79.2 \%)$ patients were in the easy airway group, according to the Cormack-Lehane classification. The two groups were then compared with each other in terms of basic patient characteristics, modified Mallampati score, anthropometric measurements of the head and neck and ULBT (Table 1). Patients in the difficult airway group were significantly younger than those in the easy airway group ( $\mathrm{p}=0.028$ ). In the difficult airway group, $80 \%$ of the patients had MMT grade $3-4(\mathrm{p}=0.001)$ while $50 \%$ of the patients were classified as ULBT $3(\mathrm{p}=0.000)$. Among the anthropometric measurements, interincisor distance (ID), hyomental distance (HMD), distance from ear tragus to the corner of the mouth (DTM) and distance from frontal plane to chin (DFC) were significantly different between the two groups $(\mathrm{p}<0.05)$.

\section{Receiver operating characteristic (ROC) analysis of predictive tests}

MMT, ULBT and four anthropometric measurements (ID, HMD, DTM and DFC), which were significantly different between the difficult and easy airway groups in univariate analysis, were directed to ROC curve analysis to evaluate their diagnostic abilities for predicting difficult airways (Figure 1). Among these tests, ULBT had the largest area under the curve ( $\mathrm{AUC}=0.880$ ) whereas ID had the smallest AUC (0.738). ULBT had a sensitivity of $83 \%$ and a specificity of $93 \%$ to predict difficult airways.

\section{Discussion}

The present study showed that MMT, ULBT and anthropometric measurements including ID, HMD, DTM and DFC were useful in predicting difficult airways in pediatric patients. According to the ROC curve analysis, ULBT was 
Table 1. Comparison of basic data and predictive tests between the two groups.

\section{Difficult airway group}

$(n=10)$

$4 \pm 3.2(1-12)$

$3(30 \%) / 7(70 \%)$

$14 \pm 4.9(4-21)$

$90.5 \pm 19.1(53-116)$

Height $(\mathrm{cm})$

MMT

MMT 1-2

MMT 3-4

ULBT

ULBT 1-2

ULBT 3

NC

ID

TMD

SMD

HMD

DTM

DFC
$1(10 \%)$

$8(80 \%)$

$1(10 \%)$

$5(50 \%)$

$26.2 \pm 1.6(24-29)$

$2.4 \pm 0.3(2-3)$

$4.6 \pm 1.2(2.5-6)$

$8 \pm 1.8(5-11)$

$3.3 \pm 0.9(1.5-4)$

$8.6 \pm 1.6(6-11)$

$13.6 \pm 1.3(11-16)$
Easy airway group

$(n=38)$

p

$6.6 \pm 3.6(1-14)$

0.028

$18(47.3 \%) / 20(52.7 \%)$

0.478

$24.5 \pm 13.7(9-65)$

0.028

$116.7 \pm 23.3(80-165)$

0.017

0.001

$23(60.5 \%)$

$6(15.7 \%)$

0.000

$25(65.7 \%)$

$2(5.3 \%)$

$27.1 \pm 4.9(11-36)$

0.087

$3.2 \pm 1.1(1-6)$

0.026

$5.6 \pm 1.8(2-10)$

0.092

$9.7 \pm 2.8(5-16)$

0.087

$4.7 \pm 1.1(3-8)$

0.001

$10.2 \pm 3.2(7-28)$

0.030

$15.5 \pm 3.7(10-34)$

0.021

Data are presented as mean \pm standard deviation for age, weight, height and seven anthropometric measurements of the head and neck; number (percentage) for other variables. $\mathrm{cm}$ : centimeter, DFC: distance from frontal plane to chin, DTM: distance from ear tragus to the corner of the mouth, F: female, HMD: hyomental distance, ID: interincisor distance, kg: kilogram, M: male, MMT: modified Mallampati test, NC: neck circumference, SMD: sternomental distance, TMD: thyromental distance, ULBT: upper lip bite test, y: year. 


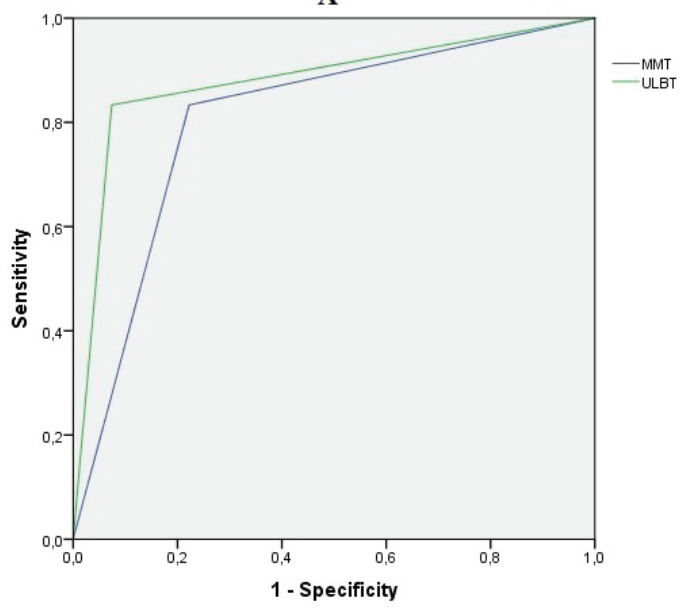

B

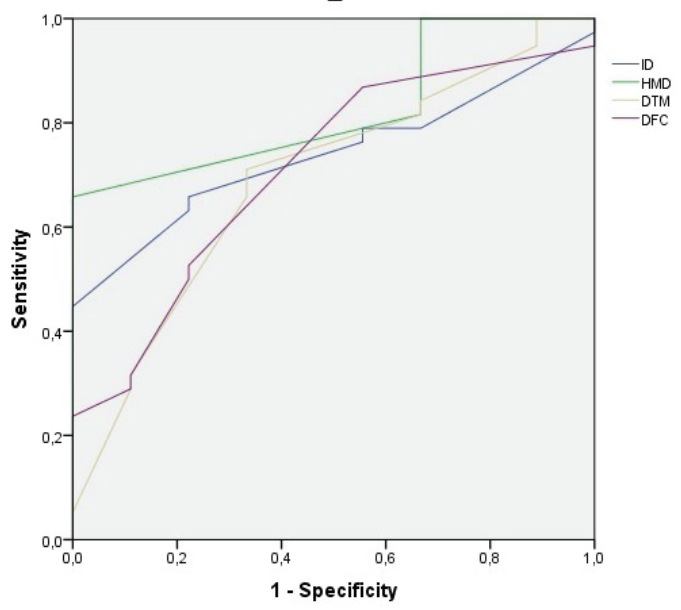

Figure 1. ROC analyses of diagnostic tests for predicting difficult airways.

A. MMT (sensitivity of $83 \%$, specificity of $78 \%$, AUC of $0.806,95 \%$ confidence interval 0.60 to $1.00, p=0.021$ ) ULBT (sensitivity of $83 \%$, specificity of $93 \%$, AUC of $0.880,95 \%$ confidence interval 0.69 to $1.00, p=0.004$ )

B. ID (sensitivity of $65 \%$, specificity of $78 \%$, AUC of $0.738,95 \%$ confidence interval 0.59 to $0.88, p=0.028$, with a cut-off value of $\leq 2.6 \mathrm{~cm}$ )

HMD (sensitivity of $65 \%$, specificity of $100 \%$, AUC of $0.825,95 \%$ confidence interval 0.70 to $0.94, p=0.003$, with a cut-off value of $\leq 4.2 \mathrm{~cm}$ )

DTM (sensitivity of $65 \%$, specificity of $67 \%$, AUC of $0.692,95 \%$ confidence interval 0.50 to $0.88, p=0.077$, with a cut-off value of $\leq 9.7 \mathrm{~cm}$ )

DFC (sensitivity of $52 \%$, specificity of $78 \%$, AUC of $0.711,95 \%$ confidence interval 0.53 to $0.89, p=0.052$, with a cut-off value of $\leq 14.2 \mathrm{~cm}$ )

found to have the strongest predictive ability compared with the other tests.

In routine anesthesia practice, difficulty in airway control is not a rare situation, with a reported incidence of up to $20 \%{ }^{[15]}$ The wide range of incidence rates in the current literature are mainly associated with different patient characteristics and different definitions of difficult intubation. The relatively higher incidence in the present study may be explained with the referral center of our hospital. The fact that $13 \%$ of respiratory problems are related to difficult intubation in pediatrics clearly shows the importance of accurate preanesthetic determination of difficult airway status. ${ }^{[1]}$
Contrary to adults, a limited number of studies have attempted to identify the effectiveness and usefulness of different airway assessments or scoring systems for the prediction of difficult airways in the pediatric population. ${ }^{7-}$ ${ }^{11,16]}$ In routine anesthesia practice, some observational parameters such as head extension width, anatomical shape of the mandible (especially degree of prognation) and tongue thickness are widely used to predict difficult airways. ${ }^{[17]}$ However, there is a lack of data regarding the association between certain anatomical measurements of the head and neck and difficult airway status in anesthetized children. Mansano et al. ${ }^{[1]}$ found that large neck circumference 
was not associated with difficulty in laryngoscopy, consistent with the result obtained from our study. However, the authors demonstrated a positive correlation between laryngoscopic difficulty and interincisor, thyromental and sternomental distances. Except for thyromental and sternomental distances, other results were in line with the findings obtained from our study. Of note, the mentioned study was only conducted on patients under 12 years old. Considering the possible differences in anatomical structures in younger children, different age distribution may be a factor affecting the statistical results. Besides those measurements, we found that HMD was another factor for predicting difficult airways. The relationship between HMD and difficult airway status was validated for adults in previous work. ${ }^{[18]}$ However, to the best of our knowledge, there is no data regarding that correlation in the literature.

As known, mandibular mobility and teeth structure play an important role in intubation. The ULBT defined by Khan et al. ${ }^{[12]}$ is based on these anatomical factors and is widely preferred due to its rapid applicability and simplicity. The authors showed that the ULBT had higher specificity and accuracy than the MMT, without a significant difference in sensitivity, positive and negative predictive values. Hester et al. ${ }^{[19]}$ also reported similar results indicating a higher predictive value of ULBT compared with MMT. In another study, ULBT showed higher specificity and positive predictive value, but not sensitivity, than TMD. ${ }^{[5]}$ Contrary to those studies, Eberhart et al. [20] demonstrated that both ULBT and MMT were poor predictors of difficult intubation. In our study, ULBT had the largest AUC in ROC curve analysis compared to other predictive tests. However, it should be noted here that 12 patients among the 15 patients in whom ULBT could not be performed in our study population were under 6 years old, indicating an important limitation for the applicability of ULBT in younger children. This situation is also valid for MMT. This is why all patients in whom MMT could not be performed were under five years old. Nevertheless, MMT had one of the highest AUC with high sensitivity and specificity rates. This finding was consistent with the results reported in the literature. For instance, Santos et al. ${ }^{[9]}$ showed a significant correlation between MMT and Cormack-Lehane index. However, the authors reported that MMT was applicable in children above 4 years old. In another study conducted with adult patients, the combination of MMT and TMD was found to be effective in predicting the incidence of difficult intubation. ${ }^{[21]}$ Similar to our study, Inal et al. ${ }^{[10]}$ demonstrated that both MMT and ULBT may be useful in pediatric patients aged 5 to 11 for predicting difficult intubation.

There were several limitations in the present study. First, the fact that it was a single-center study may limit the generalizability of the statistical results. A relatively small number of patients may be considered as a second limitation, which makes it difficult to interpret subgroup findings. A multicenter or large scale study may also be required to evaluate possible cut-off values for anthropometric measurements. However, its prospective nature and standard anesthetic protocols were the strengths of this study.

\section{Conclusion}

This study showed that ULBT, MMT, HMD and ID were the most consistent predictors of difficult airway status. ULBT was superior to other tests because of its larger AUC and higher sensitivity and specificity rates. However, difficulty in applicability in young children seemed to be the most important limitation of both ULBT and MMT.

\section{Acknowledgement: None.}

Ethics Committee Approval: The study protocol was approved by the Eskişehir Osmangazi University Clinical Research Ethics Committee (Approval number: 2019-48).

Informed Consent: All participants accepted to participate in the study and written and verbal informed consent was obtained from the parents of each child.

Author Contributions: Designing the study - Y.K., A.B., B.B.Y.; Collecting the data - Y.K., M.O., D.Ç., A.B., B.B.Y.; Analysing the data - Y.K., M.O., D.Ç., A.B., B.B.Y.; 
Writing the manuscript - Y.K. and B.B.Y.; Confirming the accuracy of the data and the analyses - M.O., D.Ç.

Conflict of Interest: The authors have no conflicts of interest to declare.

\section{References}

1. Tay CL, Tan GM, Ng SB. Critical incidents in paediatric anaesthesia: an audit of 10000 anaesthetics in Singapore. Paediatr Anaesth 2001;11:711-8.

2. Mamie C, Habre W, Delhumeau C, Argiroffo CB, Morabia A. Incidence and risk factors of perioperative respiratory adverse events in children undergoing elective surgery. Paediatr Anaesth 2004;14:218-24.

3. Weiss M, Engelhardt T. Proposal for the management of the unexpected difficult pediatric airway. Paediatr Anaesth 2010;20:454-64.

4. Samsoon GL, Young JR. Difficult tracheal intubation: a retrospective study. Anaesthesia 1987;42:487-90.

5. Salimi A, Farzanegan B, Rastegarpour A, Kolahi AA. Comparison of the upper lip bite test with measurement of thyromental distance for prediction of difficult intubations. Acta Anaesthesiol Taiwan 2008;46:61-5.

6. Min JJ, Kim G, Kim E, Lee JH. The diagnostic validity of clinical airway assessments for predicting difficult laryngoscopy using a grey zone approach. J Int Med Res 2016;44:893-904.

7. Tokmakoğlu M, Çağlar S, Ünlü S. The comparison of Mallampati and Cormack-Lehane test in prediction of difficult intubation in children. [Article in Turkish] T Clin J Med Sci 2002;22:484-6.

8. Nikhar SA, Grover VK, Mathew PJ. Predictors of intubation in children. Indian J Pediatr 2010;77:1392-4.

9. Santos AP, Mathias LA, Gozzani JL, Watanabe M. Difficult intubation in children: applicability of the Mallampati index. Rev Bras Anestesiol 2011;61:156-8.

10. Inal MT, Memiş D, Sahin SH, Gunday I. Comparison of different tests to determine difficult intubation in pediatric patients. [Article in Portuguese] Rev Bras Anestesiol 2014;64:391-4.

11. Mansano AM, Módolo NS, Silva LM, et al. Bedside tests to predict laryngoscopic difficulty in pediatric patients. Int J Pediatr Otorhinolaryngol 2016;83:63-8
Financial Disclosure: The authors declare that this study has received no financial support.

12. Khan ZH, Kashfi A, Ebrahimkhani E. A comparison of the upper lip bite test (a simple new technique) with modified Mallampati classification in predicting difficulty in endotracheal intubation: a prospective blinded study. Anesth Analg 2003;96:595-9.

13. Cormack RS, Lehane J. Difficult tracheal intubation in obstetrics. Anaesthesia 1984;39:1105-11.

14. Aktas S, Atalay YO, Tugrul M. Predictive value of bedside tests for difficult intubations. Eur Rev Med Pharmacol Sci 2015;19:1595-9.

15. Etezadi F, Ahangari A, Shokri H, et al. Thyromental height: a new clinical test for prediction of difficult laryngoscopy. Anesth Analg 2013;117:134751.

16. Mirghassemi A, Soltani AE, Abtahi M. Evaluation of laryngoscopic views and related influencing factors in a pediatric population. Paediatr Anaesth 2011;21:663-7.

17. Frei FJ, Ummenhofer W. Difficult intubation in paediatrics. Paediatr Anaesth 1996;6:251-63.

18. Huh J, Shin HY, Kim SH, Yoon TK, Kim DK. Diagnostic predictor of difficult laryngoscopy: the hyomental distance ratio. Anesth Analg 2009; 108:544-8.

19. Hester CE, Dietrich SA, White SW, Secrest JA, Lindgren KR, Smith T. A comparison of preoperative airway assessment techniques: the modified Mallampati and the upper lip bite test. AANA J 2007;75:177-82.

20. Eberhart LH, Arndt C, Cierpka T, Schwanekamp J, Wulf H, Putzke C. The reliability and validity of the upper lip bite test compared with the Mallampati classification to predict difficult laryngoscopy: an external prospective evaluation. Anesth Analg 2005;101:284-9.

21. Kandemir T, Şavlı S, Ünver S, Kandemir E. Sensitivity of the Combination of Mallampati Scores with Anthropometric Measurements and the Presence of Malignancy to Predict Difficult Intubation. Turk J Anaesthesiol Reanim 2015;43:7-12.

This is an open access article distributed under the terms of the Creative Commons Attribution-NonCommercial-NoDerivs 3.0 Unported (CC BY- NC-ND3.0) Licence (http://creativecommons.org/licenses/by-nc-nd/3.0/) which permits unrestricted noncommercial use, distribution, and reproduction in any medium, provided the original work is properly cited.

Please cite this article as: Kılıç Y., Onay M., Çetinkaya D., Bilir A., Büyükkıdan Yelken B. Comparison of different predictive tests for difficult airways in pediatrics. ENT Updates 2020;10(3): 402-408 